to have stumbled accidentally across these tar pools, but we suggest that some animals were deceived by and attracted to the pits by the strong reflection-polarization of the oil surface mimicking a body of water.

\section{Gábor Horváth*}

Biophysics Group,

Department of Atomic Physics,

Lorand Eotvos University,

H-1088 Budapest, Puskin u. 5-7, Hungary Jochen Zeil*

Kuwait University, Faculty of Science, Department of Zoology, PO Box 5969, SAFAT, 13060 Kuwait

*Present addresses: Lehrstuhl für Biokybernetik, Universität Tübingen, Auf der Morgenstelle 28, D-72076 Tübingen, Ge many (G.H); Centre for Visual Sciences, RSBS, Australan many (G. H.): Centre for Visual Sciences, RSBS, Australian tralia (J.Z.).

\section{Hidden quasars reddened by dust?}

SIR - Webster et al. ${ }^{\prime}$ argue for a large population of dust-reddened quasars, on the basis of the optical-infrared colour diversity in their new sample of radio-loud quasars with flat radio spectra. If their sample is representative of the quasar population, then their results imply that optical surveys miss about $80 \%$ of quasars, and that these missing quasars could account for the observed X-ray background. We argue that there is a simple way of avoiding these radical conclusions. Their results are influenced by an additional red, optical synchrotron component peculiar to flat-spectrum radio-loud quasars, with its own resulting colour diversity caused by the range in relative contributions of the 'normal' quasar and synchrotron components.

Flat radio spectra in quasars are caused by enhancement of synchrotron emission from compact regions within a jet by relativistic beaming in the direction of its motion $^{2}$. Selecting by flat radio spectral index should therefore bias strongly in favour of quasars whose radio jets lie very close to the line of sight. In the context of Unified Schemes ${ }^{2}$ in which the jet emerges along the relatively unobscured poles of an anisotropic distribution of material, or in

Webster, R. L. et al. Nature 375, 469-471 (1995) . Antonucci, R. A. Rev. Astr. Astrophys. 31, 473-521 (1993).

3. Rieke, G. H., Lebofsky, M. J. \& Kinman, T. D. Astrophys. J. Lett. 232, 151-154 (1979).

4. Litchfield, S. J., Stevens, J. A., Ronson, E. I. \& Gear, W. K. Mon. Not. R. astr. Soc. 274, 221-234 (1995)

5. Browne, I. W. A. \& Murphy, D. W. Mon. Not. R. astr. Soc. 226, 601-628 (1987).

6. Wright, A. E., Ables, J. G. \& Allen, D. A. Mon. Not. R astr. Soc. 205, 793-808 (1983)

7. Miller, P., Rawlings, S., Saunders, R. \& Eales, S. Mon. Not. R. astr. Soc. 254, 93-110 (1992).

8. Jackson, N., Browne, I. W. A., Murphy, D. W. \& Saikia, D. J. Nature 338, 485-487 (1989).

9. Rawlings, S., Lacy, M., Sivia, D. S. \& Eales, S. A. Mon. Not. R. astr. Soc. 274, 428-434 (1995).

10. Laing, R. A., Riley, J. M. \& Longair, M. S. Mon. Not. R. astr. Soc. 204, 151-187 (1983)

which the jet itself clears material along its path, flat-spectrum quasars should be subject to very little intrinsic reddening .

It has been known for many years, however, that flat-spectrum quasars can have unusually red optical-infrared colours (for example, ref. 3). In several cases there is direct evidence from time variability that these colours are the result of a nonthermal source of red light which is superimposed on the normal blue quasar continuum (for example, ref. 4) and which is likely to be an extension of the synchrotron emission that dominates in the radio; many other studies also suggest the presence of a beamed optical-infrared component (for example, ref. 5). Indeed, many of the Webster et al. quasars are classified optically as blue stellar objects, despite their red optical-infrared colours'. This argues strongly against a reddening interpretation, but is consistent with a beamed, red synchrotron component.

Such a beamed component of optical emission may also explain the unusually large range of broad-line equivalent widths amongst flat-spectrum quasars (compare ref. 7 with Webster et al.'s Fig. 2; see also ref. 5). An observable anti-correlation of broad-line equivalent widths with $B-K$ colour is not a firm prediction of the beaming model because the combined dispersion in the equivalent widths, the unbeamed continuum slope, and the slope of the (variable) beamed component may be very large. However, Jackson et al. ${ }^{8}$ have demonstrated an anti-correlation between the dominance of flat-spectrum radio

\title{
New photosynthesis or old?
}

SIR - Greenbaum et al. ${ }^{1}$ have described the light-induced hydrogen and oxygen evolution in a mutant strain of the green alga Chlamydomonas reinhardtii lacking photosystem I (PSI). As the mutant cells consumed carbon dioxide in the light, Greenbaum et al. suggested that reduced NADPH (nicotinamide adenine dinucleotide phosphate, obligatory for carbon fixation and normally formed by PSI) was generated by the direct reduction of $\mathrm{NADP}^{+}$by pheophytin, the low-potential electron acceptor of photosystem II $(\text { PSII })^{2}$. The authors claimed "a new type of photosynthesis being performed by the PSII light reactions exclusively".

Transient photosynthesis has been reported from a Chlamydomonas mutant lacking PSI (for example, ref. 3), as has direct reduction of $\mathrm{NADP}^{+}$by pheophytin in various PSII-enriched preparations (for example, refs 4,5 ). The quantum yields were low in the latter case ${ }^{6}$. Apparently, the forward electron transfer from the reduced pheophytin to $\mathrm{NADP}^{+}$was not competitive with its backreaction (occurring in a few nanoseconds ${ }^{7}$ ) with the oxidized primary electron donor of PSII. The emission and the equivalent widths of the emission lines. This is strong evidence for an additional source of continuum emission in flat-spectrum quasars which, if red (as expected for synchrotron emission), provides a simple explanation for the colour diversity reported by Webster et al.

Some quasars may be reddened by the mechanism favoured by Webster et al. The well-known population of red quasars associated with steep-spectrum radio sources, in which the red beamed optical component is not expected to dominate, have broad lines sometimes only revealed by near-infrared spectroscopy'. Nevertheless, complete surveys at low radio frequencies ${ }^{10}$, which are insensitive to beamed objects, contain few red quasars, again suggesting that the incompleteness in optical quasar surveys is much less dramatic than concluded by Webster et al.

Perhaps a more pertinent question is whether narrow-line objects constitute a population of obscured quasars. Studies of steep-spectrum radio sources ${ }^{2}$ are consistent with the hypothesis that narrowline radiogalaxies are quasars whose nuclei are obscured by material with column densities orders of magnitude higher than those invoked by Webster et al.

Stephen Serjeant

Astrophysics Group,

Blackett Laboratory,

Imperial College, London SW7 2BZ, UK

Steve Rawlings

Department of Astrophysics,

Nuclear Physics Laboratory,

Oxford OXI 3RH, UK

experiments with the PSI-lacking mutants, however, yielded a high rate of carbon dioxide consumption, comparable to that in wild-type cells. This high quantum yield may point to another mechanism of $\mathrm{NADP}^{+}$reduction.

The photosynthetic reaction centre of purple photosynthetic bacteria is evolutionarily and functionally related to $\mathrm{PSII}^{8}$ (except for its inability to oxidize water). Bacteriopheophytin, with the same low redox potential as its counterpart in PSII $^{9}$, does not directly reduce nicotinamide dinucleotides. Instead, they are formed by reversed electron flow through the NADH:ubiquinone oxidoreductase complex. The driving force of the reversal consists of the scalar reducing potential of the ubiquinone/ubiquinol redox pair plus the electrochemical potential difference of the proton across the photosynthetic membrane ${ }^{10}$. Chlamydomonas reinhardtii incorporates an active $\mathrm{NAD}(\mathrm{P}) \mathrm{H}$ :plastoquinone oxidoreductase in its thylakoid membrane ${ }^{11,12}$. Thus, we propose a more traditional interpretation of the data by Greenbaum et al., namely, that electrons provided by PSII first reduce the plasto- 